\title{
PAPILLARY TUMOURS OF THE CHOROID PLEXUS
}

\author{
BY \\ A. T. SANDISON \\ From the Departments of Pathology, the University, the Royal Hospital for Sick Children, and the Western \\ Infirmary, Glasgow
}

(RECEIVED FOR PUBlication DECEMBER 6, 1955)

Papillary tumours of the choroid plexus are rare. Cushing (1932) found only 12 instances in a total series of 2,023 cases of verified intracranial tumours.

At the Royal Hospital for Sick Children, Glasgow, an examination of the archives covering a period of 70 years revealed only two examples of choroidal plexus tumours in over 9,700 necropsies. These were papillary tumours of benign appearance. In this period at least 47 other intracranial neoplasms were observed. The majority of these were astrocytomata of varying degrees of differentiation, although ependymoma and medulloblastomas are also recorded.

The records of 14,000 necropsies at the Western Infirmary, Glasgow, carried out in the past 55 years, showed only two cases of papillary tumour of the choroid plexus and in one no histological examination was made. In a third case, regarded as a highly cellular tumour of choroid plexus origin, re-examination suggests that it was, in fact, probably a metastatic carcinoma of bronchial origin.

\section{Case Reports}

Case 1.-The patient, a girl aged 14 months, was transferred to the wards of Dr. J. H. Hutchison at the Royal Hospital for Sick Children, Glasgow, from a peripheral hospital where she had been diagnosed as suffering from lymphocytic meningitis with hydrocephalus. Investigation of the cerebrospinal fluid after admission showed only a high protein content. The optic discs were seen to be blurred, and radiological examination showed hydrocephalus. Twentyone days later the child went into status epilepticus, developed hyperpyrexia $\left(107^{\circ} \mathrm{F}\right.$.), and died within a few hours.

Necropsy Findings. - The body was that of a female child weighing $8 \mathrm{~kg}$., of appearance consistent with the stated age. The cranial circumference was $46.0 \mathrm{~cm}$. and the fontanelles were large, open, and tense. On dissection there was no abnormality of the mouth, pharynx, oesophagus, stomach, small and large intestine, including the rectum and anus. The liver was of normal contour, consistency, and colour, and the gallbladder, bile ducts, and pancreas were normal. The spleen was pale and firm, and the mesenteric nodes pale and more prominent than usual. The trachea, bronchi, lungs, and pulmonary vessels showed no features of note, nor did the pericardium, heart, or great vessels. The kidneys, ureters, bladder, uterus, tubes, and ovaries were normal, as were the thyroid, thymus, adrenal, and pituitary. Histological examination of those organs showed no features of interest, apart from some congestion and fatty change in the liver and reactive hyperplasia in the spleen and lymph nodes.

The spinal column and spinal cord appeared normal. The cranial bones were expanded and much thinned out, and the floor of the anterior fossa was finely granular. The brain $(1,400 \mathrm{~g}$.) showed obvious hydrocephalus on removal, the right hemisphere being larger than the left. The gyral pattern of the right parietal and occipital lobes was well developed, but over the rest of the cerebrum the gyri were narrow and poorly defined. There was slight thickening of the basal leptomeninges with some ballooning over the midbrain, and the fourth ventricle was a little dilated. The brain was fixed entire and section later showed some degree of pontine hypoplasia, while lateral compression of the midbrain had narrowed the aqueduct into a vertical slit. Coronal section of the cerebrum showed dilatation of the third ventricle and both lateral ventricles, which were displaced to the left. Anteriorly the right ventricle was more dilated than the left, but posteriorly the right ventricle was occupied by a large tumour mass measuring 7.0 by 6.0 by $6.0 \mathrm{~cm}$. (Figs. 1 and 2). This mass was covered by a thin, semi-translucent membrane and had a smoothly rounded anterior pole. Although attached by a pedicle to the choroid plexus, it was nowhere adherent to the ventricular wall. Section showed this mass to be a pale pinkish-brown, finely granular papillary tumour which included many areas of microscopic calcification. The appearances were typical of a so-called benign papillary tumour of the choroid plexus.

Histological Examination.-Histological examination confirmed the morbid anatomical diagnosis. The tumour was well differentiated, cytologically benign, and capsulated. No evidence of invasiveness was noted. The pattern was that of innumerable, fine, well-delineated papillary fronds, and although vascular 


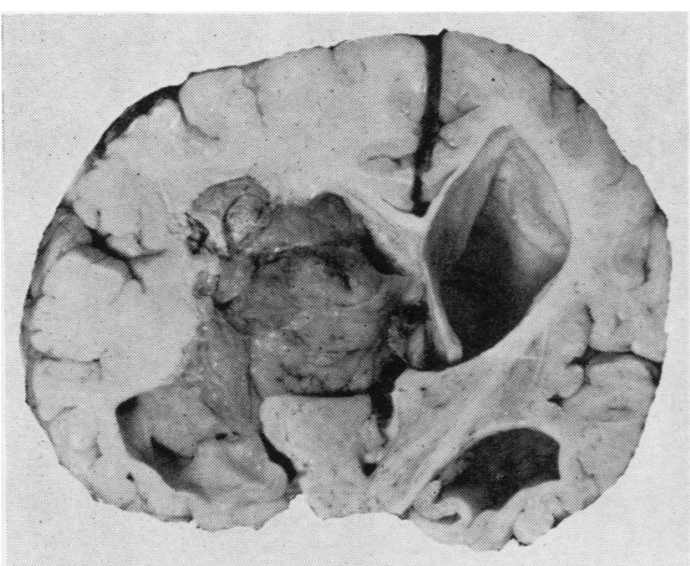

FIG. 1

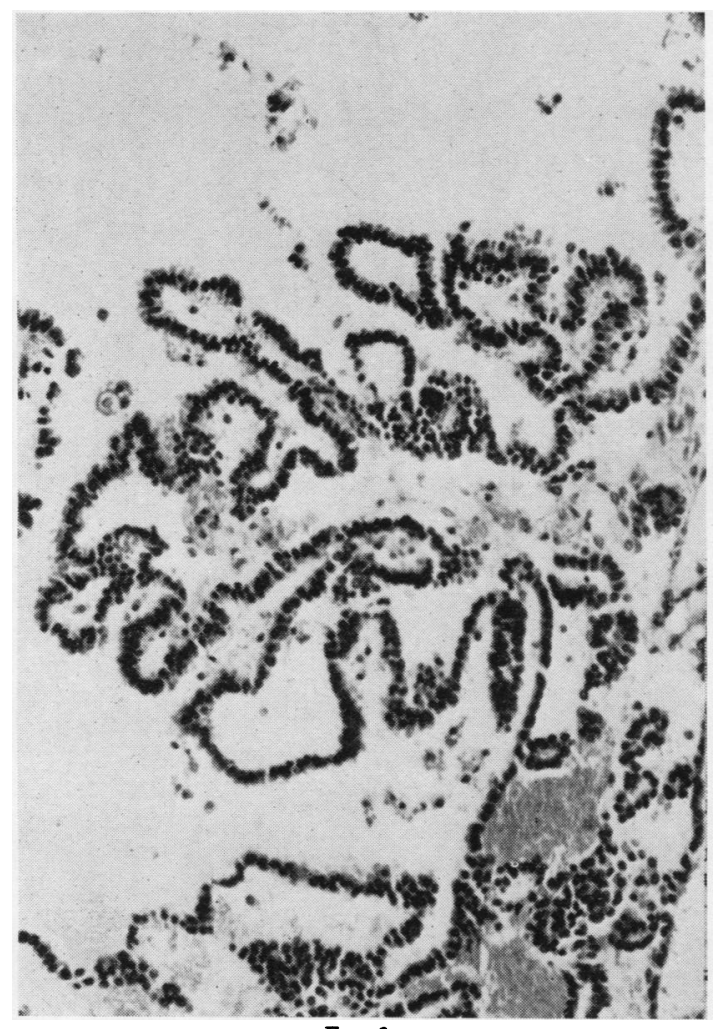

Fig. 3

Fig. 1.-Coronal section of brain of Case 1 to show ventricular dilatation and large size of tumour in right lateral ventricle.

Fig. 2,-Coronal section of right hemisphere posterior to that seen in Fig. 1. The ventricle is filled by the tumour and the cortex is much thinned out.

Fig. 3.-Typical frondose appearance of tumour which closely resembles normal choroid plexus in Case 1. Haemalum and eosin $\times 180$.

Fig. 4.-High-power view of tumour from Case 1. The tall, columnar character of the cells and compact nuclei are shown, also calcified material between the fronds $\times 570$.

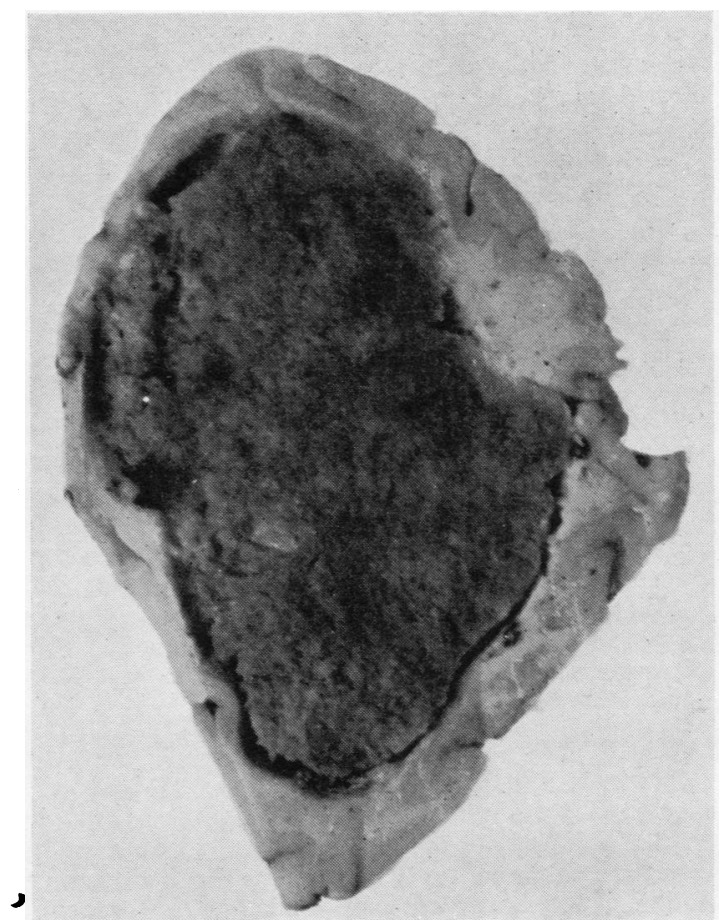

Fro. 2

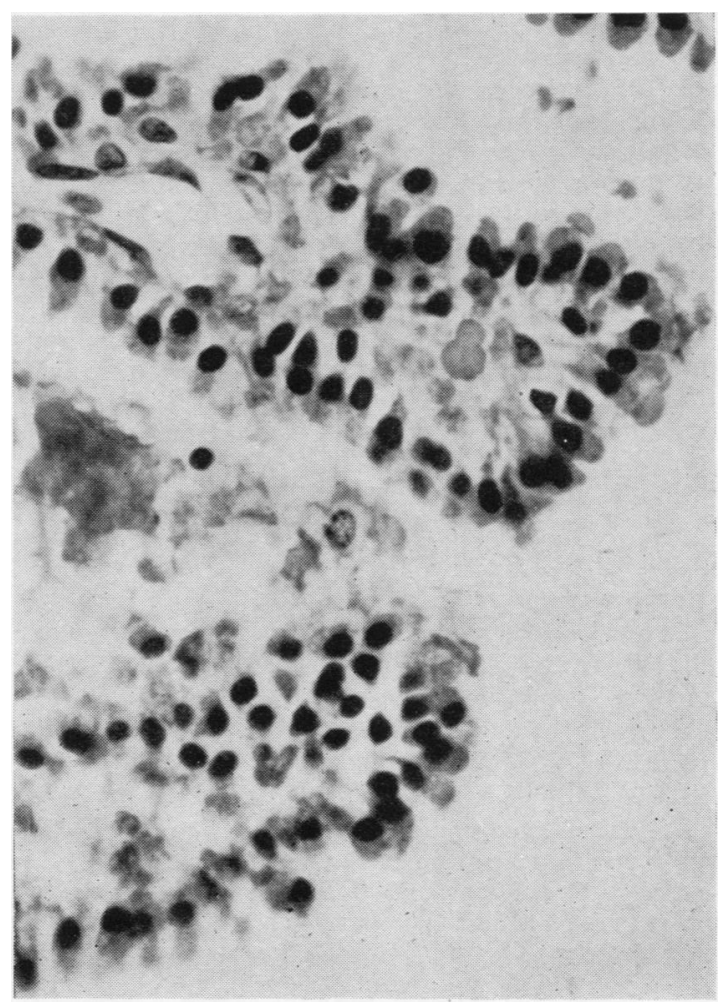

Fig. 4 
there was no evidence of haemorrhage or necrosis. The appearances were reminiscent of choroid plexus, namely, a simple frondose pattern with a columnar epithelial covering, the cells being eosinophilic and having a small, compact, darkly staining nucleus, usually situated close to the basement membrane (Figs. 3 and 4). Scattered throughout were tiny spherules of haematoxyphil calcific material arranged in simple laminae. These spherules, according to Willis (1953), are more frequently seen in papillary tumours in adults and were not seen in sections from Cases 2 and 3.

Case 2.-The patient, a 5-week-old girl, was admitted to the wards of the late Professor G. B. Fleming at the Royal Hospital for Sick Children, Glasgow. She had been a full-time, spontaneously delivered infant of $4.1 \mathrm{~kg}$. body weight. For two weeks she had been ailing, and three days before admission it was noticed that the head was enlarged and the scalp veins engorged. Lumbar puncture showed a xanthochromic fluid, under slightly increased pressure, which microscopy showed to contain numerous red blood cells, and proved sterile on culture. Ventricular puncture revealed a considerable increase in pressure.

Necropsy Findings.-The body was that of a wellnourished female infant with a somewhat enlarged head. Dissection of the thorax showed diffuse bronchopneumonia only, while in the abdomen no significant observations were made. The fontanelles were widely opened, and the brain was removed with difficulty owing to its softness. At the base, around the roof of the fourth ventricle, and over the cerebellum there was some yellowish staining, indicative of old haemorrhage, with some adhesion of the meninges. The whole ventricular system was dilated, especially in the lateral ventricles around which the cortex was greatly thinned. A mass was felt in the left lateral ventricle, and on section a tumour of papillary appearance was noted. This was attached by a stalk to the posterior end of the choroid plexus and lay freely in the ventricle at its posterior end.

Histological examination showed this to be a papillary tumour with a vascular core, closely resembling the structure of choroid plexus (Fig. 5). Some areas of infarction were noted with a moderate infiltrate of polymorphonuclear leucocytes. In these areas the nuclear outlines were lost and the cells were pinkishred. These infarcts were therefore presumably of some little duration. No calcified spherules were present.

Case 3.-The patient, a boy aged 16, was admitted to the wards of the late Mr. R. F. Young, at the Western Infirmary, Glasgow, with ascites, the result of calcific obliterative pericarditis. Partial pericardectomy was performed three weeks before death with temporary relief only.

Necropsy Findings. - The body was that of a poorly nourished boy with an infected thoracic wound. The presence of a calcified adherent pericardium was confirmed; ascites was severe and the abdominal viscera showed some chronic venous congestion. There was

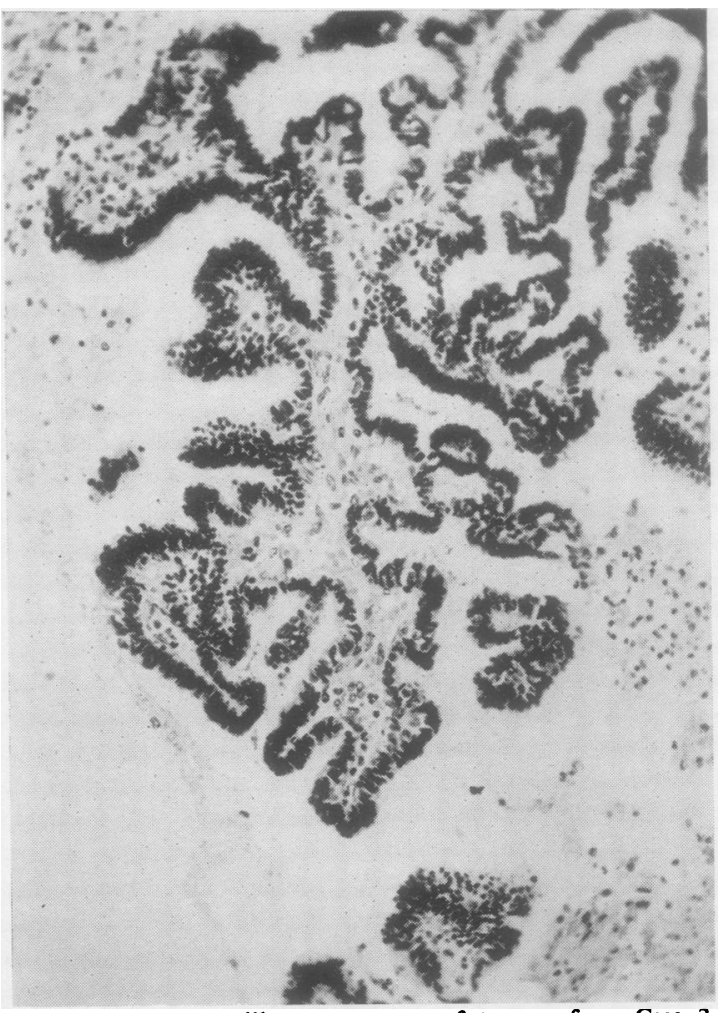

Fig. 5.-Similar papillary appearance of tumour from Case 2. Haemalum and eosin $\times 180$.

no abnormality of the cranial cavity or meninges. On section through the brain-stem a papillomatous tumour was noted projecting into the fourth ventricle from the choroid plexus. This measured $2.0 \mathrm{~cm}$. in diameter. The aqueduct and third ventricle were slightly, and the lateral ventricles rather more, dilated.

Histological examination showed the tumour to be a typical papillary tumour of the fourth ventricle with a fibrovascular core and covered by tall columnar non-ciliated epithelium of eosinophilic character and having the nuclei placed close to the basement membrane. No calcified spherules were present (Fig. 6).

Case 4.-The patient, a man aged 47, was admitted to the wards of the late Mr. A. Young at the Western Infirmary, Glasgow, five months before death, with signs of increased intracranial pressure. Radiography showed some destruction of the sella turcica. One month after admission an osteoplastic flap was turned down, but no tumour was found and the appearances at the base of the brain were thought to suggest a chronic meningitis, although culture was sterile and no tubercle bacilli were found. Terminally there was drowsiness, tachycardia, tachypnoea and hyperpyrexia.

Necropsy Findings. - The body was that of a rather emaciated, middle-aged male. The lungs were emphysematous with bronchopneumonia at the bases. 


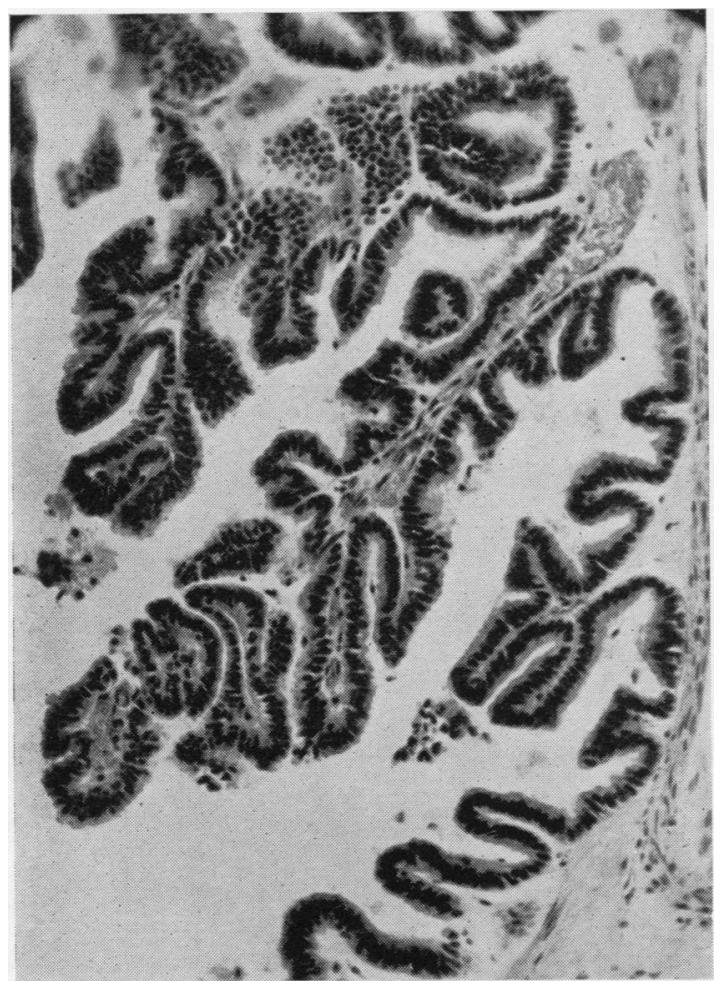

Fig. 6.-This section from Case 3 shows a very similar frondose pattern. Haemalum and eosin $\times 180$.

The liver was fatty, but the abdominal organs otherwise showed no significant change. On opening the head, the subarachnoid space was seen to contain brownish-yellow fluid, but the convolutions were not flattened. When the brain was sectioned a haemorrhagic mass was found in the posterior horn of the left lateral ventricle attached to the choroid plexus. The tumour measured about $5 \mathrm{~cm}$. across and $2.5 \mathrm{~cm}$. in its antero-posterior dimension. Much of it was haemorrhagic, but anteriorly it was whitish and more cellular.

The structures anterior to the horn were somewhat displaced forwards. The cerebrospinal fluid in the ventricles was normal in amounts but of brownishyellow colour. The brain was preserved as a museum specimen and no histological examination was made. Nevertheless it seems possible that this may have been a papillary tumour of the choroid plexus.

\section{Discussion}

Papillary tumour or papilloma of the choroid plexus, although rare, is a tumour occurring predominantly in childhood. Cushing (1932) found only 12 choroidal papillomata in a series of verified intracranial neoplasms numbering 2,023, i.e., an incidence of only $0.59 \%$. Cuneo and Rand (1952), however, encountered three cases in a series of 83 proved intracranial tumours in children. They state that this incidence of $3.66 \%$ is quite unusual ; nevertheless Bodian and Lawson (1953) also discovered three instances of choroid papilloma out of 129 cases of intracranial tumours in children.

Herren (1941) collected 88 cases from the literature including one of his own. Van Wagenen (1930) reviewed 45 cases in detail, $50 \%$ of which were situated in the fourth ventricle, $34.7 \%$ in the lateral ventricles, and $17.3 \%$ in the third ventricle. In the present series of three proved cases two were in the lateral and one in the fourth ventricle. In one case reviewed by Posey (1942) the tumour was bilateral. Van Wagenen noted that papillary tumours of the choroid plexus occur most frequently in the first decade of life, although he reviews one case occurring in the eighth decade. One case occurred in a newly born infant and it is possible that our Case 2 may have been congenital. The sexes appear to be almost equally affected.

The term "papilloma" is often applied to these tumours because their structure closely resembles that of normal choroid plexus. Because of their site of origin they may be disseminated by the cerebrospinal fluid through the ventricular system to the basal meninges and to the meninges of the spinal cord. They are not highly malignant, but because of the dissemination some authorities prefer to refer to them as "carcinomas" of the choroid plexus. Willis (1953), however, emphasizes that separation into benign and malignant growths is quite arbitrary and serves no useful purpose. He points out also that papillary tumours of the choroid plexus may occur in animals. The tumours are frequently vascular, friable, obviously papillary, and sometimes gritty from granular calcification as in Case 1. They vary considerably in size from small circumscribed tumours to huge masses occupying much of the ventricular system. Histologically the majority have a fully differentiated choroid-plexus-like structure. Not infrequently the lateral ventricular tumours are surrounded by a thin membrane of connective tissue as in Case 1. Turner and Simon (1937) report two cases in which mitotic figures and multinucleation of cells led to a diagnosis of malignancy. Willis rightly stresses, however, that marked anaplasia in a supposed choroid plexus tumour requires consideration of the possibility of its being metastatic from a visceral glandular carcinoma.

$\mathrm{He}$ cites secondary tumours of the choroid plexus as having been recorded from lympho[sarcoma, melanoma, thymona, carcinoma of the 
pancreas, carcinoma of the thyroid and, probably most frequently, from carcinoma of the bronchus (Willis, 1952).

Rarely surgical removal has been successful, but recurrence is frequent. Cuneo and Rand (1952) cite Sachs in 1942 as claiming a six-year cure after removal of a papilloma of the fourth ventricle, and Walker and Horrax in 1947 a survival of 10 years in an adult where a papilloma of the fourth ventricle required three operations because of recurrence. Van Wagenen (1930) succeeded in totally removing a papilloma of the left lateral ventricle in a 3-month-old infant. Radiotherapy may diminish the tumour size, but never effects a cure.

The cerebrospinal fluid may be xanthochromic as in Cases 2 and 4. Internal hydrocephalus may be produced, sometimes unilaterally, and clinical diagnosis is difficult. Headache, nausea, and vomiting are frequently present. Ventriculography may outline the tumour. If sudden haemorrhage occurs from the tumour the clinical picture may be indistinguishable from spontaneous subarachnoid haemorrhage

\section{Summary}

Three cases of proved papillary tumour of the choroid plexus occurring in children are described and one other choroidal tumour in an adult is discussed briefly. Some aspects of the pathology and clinical findings in this condition are outlined.

I am grateful to Professor D. F. Cappell and Dr. A. M. MacDonald for access to the necropsy reports of the Western Infirmary and Royal Hospital for Sick Children, Glasgow, and to Dr. J. H. Hutchison for permission to publish Case 1. Mr. N. Russell gave technical assistance and Mr. W. Mason took the photographs.

\section{REFERENCES}

Bodian, M., and Lawson, D. (1953). Brit. J. Surg., 40, 368.

Cuneo, H. M., and Rand, C. W. (1952). Brain Tumors of Childhood. Thomas, Springfield, Illinois.

Cushing, H. (1932). Intracranial Tumours. Thomas, Springfield,

Illinois.
Herren, R. Y. (1941). Arch. Surg. (Chicago), 42, 758.

Posey, L. C. (1942). Arch. Path. (Chicago), 34, 911.

Turner, O. A., and Simon, M. A. (1937). Amer. J. Cancer, 30, 289.

Van Wagenen, W. P. (1930). Arch. Surg. (Chicago), 20, 199.

Willis, R. A. (1952). The Spread of Tumours in the Human Body, 2nd ed. Butterworth, London.

(1953). Pathology of Tumours, 2nd ed. Butterworth, London. 